\title{
Improving Guide Dog Team Play with Accessible Dog Toys
}

\author{
Sabrina Hauser \\ School of Interactive Arts \\ \& Technology \\ Simon Fraser University \\ Surrey, British Columbia, Canada \\ shauser@sfu.ca
}

\section{Ron Wakkary}

School of Interactive Arts

\& Technology

Simon Fraser University

Surrey, British Columbia, Canada

rwakkary@sfu.ca

\section{Author Keywords}

Human-Canine Interaction; Visually Impaired; Guide Dogs; Interaction Design.

\section{ACM Classification Keywords}

H.5.m. Information interfaces and presentation (e.g., HCI):

Miscellaneous.

Permission to make digital or hard copies of part or all of this work for personal or classroom use is granted without fee provided that copies are not made or distributed for profit or commercial advantage and that copies bear this notice and the full citation on the first page. Copyrights for third-party components of this work must be honored. For all other uses, contact the owner/author(s). Copyright is held by the author/owner(s). CHI 2014, April 26-May 1, 2014, Toronto, Ontario, Canada. ACM 978-1-4503-2474-8/14/04.

http://dx.doi.org/10.1145/2559206.2581316

\begin{abstract}
People with vision impairment have been a longstanding well-recognized user group addressed in HCI. Despite the recent interest in studying sighted dog owners and their pets in $\mathrm{HCI}$, there is a noticeable gap in the field with regards to research on visually impaired owners and their dogs (guide dog teams). This paper presents portions of an ongoing study that explores interactions of guide dog teams revealing major opportunities for focusing on challenges faced in "off-work" everyday activities. In particular, opportunities point to promoting design interventions enriching play-interaction through accessible dog toys utilizing sensor technologies.
\end{abstract}

\section{Introduction}

$\mathrm{HCI}$ research and industry have recognized the visually impaired as a user group for decades $[1,6,8,9,10,16]$. Accessible technology developments such as tools, applications and gadgets have been improving the lives of people challenged with visual impairment, enabling them to be more independent. One of the most common challenges experienced by people who are blind is their independence while traveling. Different mobility aids have been developed to enhance their travel abilities. The most popular travel aid is the long cane.

Nevertheless, research has shown that guide dogs are the most beneficial travel aid because they provide safe guidance when traveling $[11,23]$ and increased mobility and independence, while offering the additional benefit of companionship. A guide dog owner, however, like any 
other dog owner has to provide for the physical and emotional needs of a dog. Dogs need food, several opportunities each day to relieve themselves, grooming, veterinarian visits, playtime and affection [5].

Recently, the study of sighted dog owners and their pets has gained interest in HCI. Many studies have looked at computer-mediated human-pet interaction (e.g.

$[3,7,18,26])$ mainly focusing on connecting humans and their pets when apart. As an emerging field, animal- and pet-focused research still remains understudied in $\mathrm{HCI}$ $[14,15,18]$. Studies from disciplines outside of HCI have looked at guide dog teams. This body of work concerns itself primarily with the impact on identities of guide dog owners [19], the benefits of guide dogs [11,23], and the experience of their usage [24]. However, research on technology and guide dog teams is conspicuously absent across intersecting disciplines.

Our approach attempts to bring these different strands together by exploring new ways in which technology could positively enhance the everyday lives and interactions of guide dog teams in the future. In this paper we present findings from an ongoing study of twelve guide dog teams, which reveal opportunities for $\mathrm{HCI}$ and design research. We describe significant challenges guide dog teams face in their play and propose a research agenda for supporting play interactions with accessible dog toys.

\section{Related Work in HCI}

An increasing body of research has been looking at canine-related HCI. Focusing on human-canine interactions between people and their pet dogs, Paasovaara et al. [18] developed the 'paw tracker concept', which combines sensor-based dog created content with social media to connect a dog that stays home with a human who is working, enabling the human to check on the pet on an automatically updated blog. Similarly, Hu et al. [14] and Yonezawa et al. [26] introduce prototypes for remote pet control (or humancomputer-animal interfaces), in one case combined with social media [26]. Neustaedter and Golbeck [17] investigate the design of systems for pet video chat for human-dog interactions. Wingrave et al. [25] introduced game-prototypes for strengthening the human-animal bond of dog owners and their dogs. Mancini et al. [14] explored how tracking technologies can reconfigure the relationships between humans and canines. Moreover, Weilenmann and Juhlin [22] conducted a study observing hunters using GPS devices to monitor their dogs to find out how technology assists the interactions of those human-canine teams.

A new emerging field with an interesting overlap has recently been recognized in the HCI community: Animal-Computer Interaction (ACI). Mancini provided an overview of ACI discussing key issues such as ethics and benefits pointing out that ACI could inform, "the design of technology that enables the animals we live and sometimes work with to effectively communicate with us, increase their participation in our interactions, and constructively influence our environments" [13 p.72]. This can be related to human-guide dog interaction, since those dogs work for their owners. Clearly, ACI offers much potential and will increasingly become a part of discussions within the HCI community (see Mancini $[12,13])$.

In describing related works within $\mathrm{HCI}$ we see how research on technology and guide dog teams is absent. Our approach attempts to situate prior research on the relationship between dogs and owners in guide-dog teams within the purview of $\mathrm{HCI}$, and by proposing new 


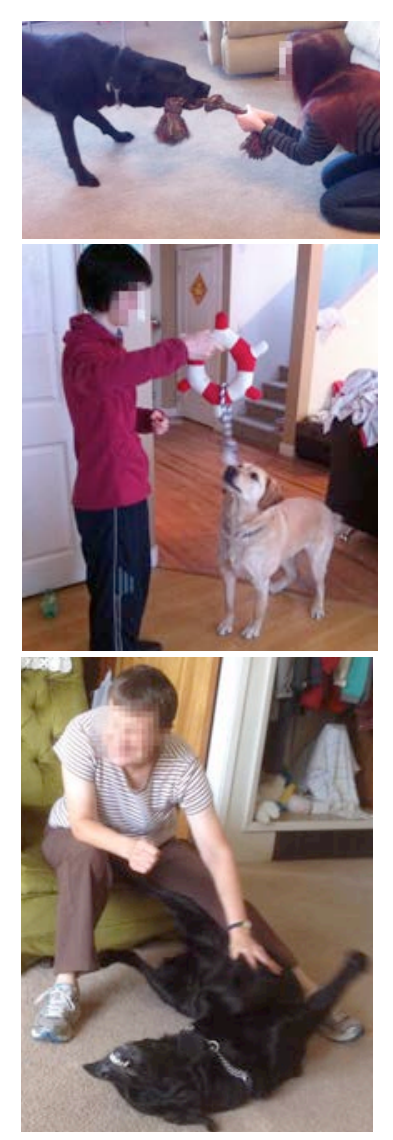

Figure 1 Play-Interactions in Guide Dog Teams

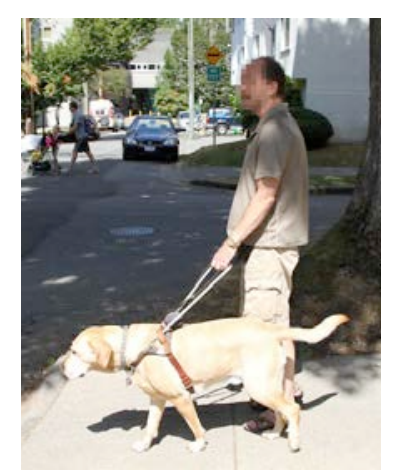

Figure 2 Work-Interaction in a Guide Dog Team ways technology could positively enhance the everyday lives and interactions of these teams in the future.

\section{Studying Guide Dog Team Interactions}

In order to gain a rich understanding of the interactions of guide dog teams, we interviewed and observed twelve participants - eight were female and four male. They all lived in or close to a major metropolitan city in Canada, were aged 21 to 67 , and used a guide dog. Five participants were early blinds, meaning they had been blind since or early after birth. Seven participants were late blinds, they lost their sight later in life.

Guide dogs are trained for two years, work for approximately eight years and then retire. Three participants were partnered with their first guide dog, four participants were with their second, two with their third, four with their fourth and one was with her fifth guide dog. The length of guide dog ownership ranged from one and a half to over 30 years. The dogs came from three different guide dog schools: BC Guide Dogs (BC, Canada), The Seeing Eye (NJ, US), and Guide Dogs for the Blind (CA and OR, US).

We conducted semi-structured interviews in the participants' homes. Questions were about the guide dog handlers, the dogs and their life, exploring routines, tasks, activities, play and challenges. Moreover, questions targeted the use of and relationship to technology. The observations in our study explore interactions of guide dog teams both in the home and outside. In order to get a detailed understanding of their practices and routines we spent between 2.5 and 4.5 hours with each team, depending on their willingness and comfort. In the following two sections we share a sample of our findings.

\section{Work vs. Play}

An important observation segmented interactions between guide dog teams into two main scenarios: work and off work. A clear indicator for these modes is the harness, which the dog is wearing while working and not wearing while off work (see Fig.1 and 2).

\section{Guide Dog Teams at Work}

At work, guide dogs wear a harness and guide their owners (see Figure 2). In this mode, the dog could be considered an instrument in his role of a working assistance dog. When working, the interactions of guide dog teams are limited. The owner knows where to go, gives the dog commands, and monitors traffic and lights. The dog guides the visually impaired owner safely wherever s/he needs to go, around obstacles and towards steps or doors. Ideally, the guide dog stays concentrated and focused, and does not get distracted. However, dogs can become distracted; the handler watches out for this, at times refocusing the dogs attention with a command and a leash pull.

Through the working relationships guide dog owners develop an essential strong bond with their dogs, relying on them in work situations. Interestingly, we found the strength of the bond comes almost entirely through the working relationship of guide dog teams, which builds up trust, confidence and awareness through the working routines.

Off-work: Human-Pet Interactions of Guide Dog Teams When off work, the harness is taken off and guide dog teams rest, walk, play, and communicate together. The owner takes care of the dog, providing him with certain privileges that add up to a typical 'dog's life', which includes: feedings, several opportunities to go to the bathroom, grooming, attention and interaction (e.g. talking to the dog and giving him affection). 

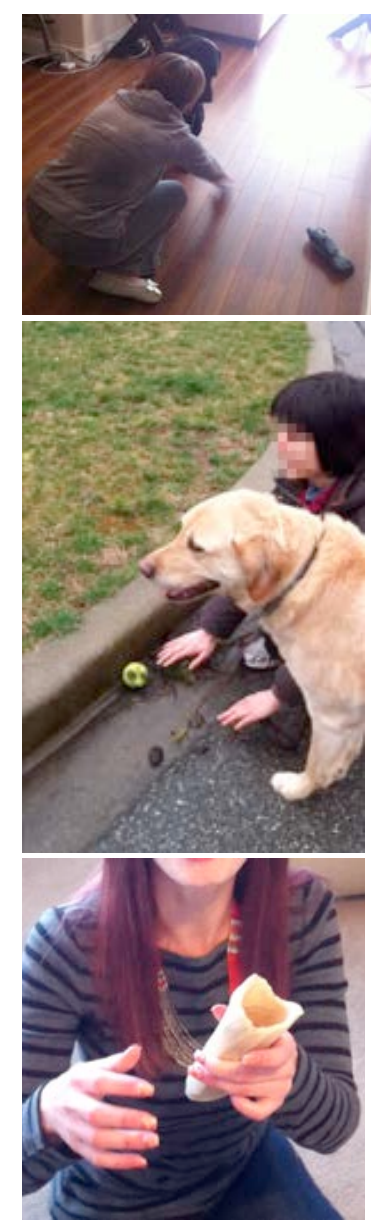

Figure 3. Challenges with dog toys: Participants $a, b$ ) search for toys and c) shows a toy with sharp edges that led to injuries
Additionally, playing and free running is important. In this mode, guide dogs are pets with individual behaviors. Similar to the routines of non-visually impaired human-canine teams, the interactions of nonworking guide dog teams can vary substantially. Some dogs are more playful and enjoy off-work time to have fun, while others may prefer to rest.

When guide dog teams are 'off work' and the dog is 'just a pet' trust, confidence and awareness present in work scenarios appeared to be largely absent.

Our findings in both work and off-work interactions offer several opportunities for further research and design interventions. In this paper we will now further discuss off-work, focusing on play-interactions, an area we found guide dog teams have significant challenges in.

\section{Challenges in Play}

Guide dogs are generally very playful, due to their breed's (mostly Labrador Retrievers) character. However, there are no special dog toys for blind dog owners and they end up using toys designed for sighted dog owners such as ropes, tug toys, balls, plush toys, squeaky toys, and bones. Our participants mentioned several challenges with dog toys.

Finding toys outdoors and indoors

It is hard to find a toy when the dog is not interested in picking it up or if the toy is out of reach for the dog (e.g. when it happens to be underneath a couch or stuck in a tree or bush). Fig.3 ( $a$ and $b$ ) shows two participants searching for dog toys that are right in front of them. We observed several searches like this.

Toys as potential hazard

Participants were unaware of the locations of some of their dog toys and they were often laying around on the floor as potential hazards for the blind owner. Some tried to keep toys away but gave their dog access to a few. We found that all participants had stepped on dog toys before and two had seriously injured themselves as a result. In one case, a participant fell down stairs in her house because of a poorly placed dog toy. In Fig.3c), one participant shows her dog's favorite toy; a bone, which she often hurts herself on by stepping on the sharp edges when walking around her apartment.

\section{Unawareness of dog behavior}

Dog owners did not notice when their dogs became bored, tired or were no longer interested in playing (e.g. they became busy with other things such as sniffing). This caused confusion for owners due to the lack of response by their dogs.

Guide dog owners are very grateful for the abilities of their dogs and want to give back to them. Play gives them an opportunity to do so. However, often described challenges get in the way. In what follows, we will suggest design interventions that address some of these issues in guide dog team play.

\section{Accessible Dog Toys}

To date, there are no digital artifacts developed specifically for guide dog team play. This forces guide dog owners to use available dog toys targeted towards sighted dog owners. The discussed challenges point to a future opportunity area of accessible dog toys utilizing sensor technologies to improve guide dog team play and enrich the experiences of guide dog owners. For example, dog toy prototypes utilizing sensortechnologies could record and communicate situational information, such as locations and distances of toys to the dog owner. Moreover, they could use auditory signals to indicate when a dog dropped a ball. Additional sensors could track the dog and communicate its location and distance to the owner through non-visual 
sensory modalities. Collectively, this could lead to enriched experiences in play within guide dog teams. In what follows, we propose a research agenda to support future work in this area.

\section{Research Agenda: Our Next Steps}

\section{Prototype Accessible Dog Toys}

As a first next step, we plan to build on the opportunity area outlined above through augmenting current dog

toys with sensors and non-visual output mechanisms in a series of prototypes.

2. Test Prototypes Studying Form, Material, \& Feedback Prototypes of accessible dog toys will be exposed to our participants in an initial round of testing. In this design process informed by users, we will assess the forms, physical materials and feedback mechanisms comprising prototypes in terms of their suitability and appropriateness for our audience. Emerging research exploring auditory feedback (e.g. [20]) and vibro-tactile interfaces (e.g. [4]) will be used to support future research.

\section{Design Iterations}

We will then iterate on our prototypes, incorporating findings from our form, material and feedback studies. The outcome will be a first series of accessible dog toys. At this point, we may include a partner from industry (e.g., dog toy manufacturers).

4. Long-term field trials with participants

In a long-term deployment study we will observe our participants using the accessible dog toys to investigate their everyday experiences of use. Beyond the toys intended use, we will pay close attention to possible emergent, unintended uses or appropriations. For instance, over time, guide dog users might become familiar with new play interactions and ad-hoc games and other experiential play-interactions could emerge. We expect that this field study will reveal new insights into guide dog team interactions.

\section{Constraints and Challenges}

When designing for guide dog teams several design constraints and challenges need to be considered.

\section{Form Factor}

When using technologies on guide dogs, the form factor of any attachments must be kept small so that the dog does not think it is in harness. This is important as the harness differentiates work time from non-work time for the dog. For example, the design of Wingraves [25] tracking prototype for dogs would not be suitable for a guide dog since its shape is very similar to a harness.

\section{Safety Issues}

Technologies implemented into toys will need to be safely protected from the dog. We will look at best practices of current interactive dog toys design for sighted human-canine teams (e.g., dogtronics, alphadogtoys, gogodogpals). Additionally, we want to explore novel solutions of addressing this issue.

\section{Ethical Issues}

More broadly, when working with animals, ethical issues need to be carefully considered. Technology should not be invasive and augmentations of animals should clearly be ethically deliberated (see also [21]). We will critically incorporate and reflect on these considerations throughout our design process. 


\section{Contribution \& Future Work}

In this paper, we explore interactions of guide dog teams sharing portions of an ongoing study. Findings from our fieldwork indicated playful interactions are not fully supported. We propose a future opportunity area of accessible dog toys utilizing sensor technologies to improve guide dog team play. This can have implications for richer experiences of guide dog owners. Additionally, routinely practiced successful play might develop and sustain trust, confidence and awareness in off-work situations. We have described the next steps of this research with an agenda on the design and research process of accessible dog toy prototypes.

This work makes two contributions. First, it advances the HCI community's understanding of guide dog teams as an important user group. Second, it details design opportunities for play interaction to help critically frame future $\mathrm{HCI}$ work in this emerging area.

\section{Acknowledgements}

We thank our participants for their input, which will continue shaping our research in this area. We thank Will Odom for helping with this work.

\section{References}

[1] Apple, Accessibility - VoiceOver.

http://www.apple.com/accessibility/voiceover.

[2] Bigham, J., Jayant, C., Ji, H., Little, G., Miller, A., Miller, R., Tatarowicz, A., White, B., White, S. and Yeh,

T. VizWiz: nearly real-time answers to visual questions. In Proc. UIST '10. ACM Press, 333-342.

[3] Britt, W., Miller, J., Waggoner, P., Bevly, D., \& Hamilton, J. An embedded system for real-time navigation and remote command of a trained canine. In Personal \& Ubiquitous Computing, 15(1), (2011),61-74. [4] Giudice, N., Palani, H., Brenner, E. \& Kramer, K. Learning non-visual graphical information using a touchbased vibro-audio interface. In Proc. ASSETS '12. ACM Press (2012), 103-110.

[5] Guide Dogs for the Blind. http://www.guidedogs.com

[6] Hersh, M., \& Johnson, M. Assistive Technology for Visually Impaired and Blind People. Springer, (2008). [7] Hu, F., Silver, D., \& Trudel, A. LonelyDog@ Home. Web Intelligence, IEEE/WIC/ACM (2007), 333-337.

[8] Humanware. Low Vision Aids for Macular Degeneration. http://www.humanware.com. [9] JAWS, Windows Screenreading Software http://www.freedomscientific.com/jaws-hq.asp.

[10] Lévesque, V. Blindness, technology and haptics. Center for Intelligent Machines, (2005).

[11] Lloyd, J., La Grow, S., Stafford, K., \& Budge, R. The Guide Dog as a mobility aid. International

Journal of Orientation \& Mobility, 1, (2008), 34-45. [12] Mancini, C. 2013. Animal-computer interaction (ACI): changing perspective on $\mathrm{HCI}$, participation and sustainability. In Proc. CHI EA '13. ACM, 2227-2236. [13] Mancini, C. Animal-Computer Interaction: A manifesto. interactions, 18(4), ACM Press (2011), 69-73.

[14] Mancini, C., Linden, J., Bryan, J. \& Stuart, A. Exploring interspecies sensemaking: dog tracking semiotics and multispecies ethnography. In Proc. UbiComp '12. ACM Press (2012), 143-152.

[15] Mankoff, D., Dey, A., Mankoff, J., \& Mankoff, K. Supporting interspecies social awareness: using peripheral displays for distributed pack awareness. In Proc. UIST '05, ACM Press (2005), 253-258.
[16] Mankoff, J., Fait, H., \& Tran, T. Is Your Web Page Accessible? A Comparative Study of Methods for Assessing Web Page Accessibility for the Blind. In Proc. CHI '05, ACM Press (2005), 41-50.

[17] Neustaedter, C. and Golbeck, J. 2013. Exploring pet video chat: the remote awareness and interaction needs of families with dogs and cats. In Proc. CSCW '13. ACM Press, 1549-1554.

[18] Paasovaara, S., Paldanius, M., Saarinen, P., Häkkilä, J., \& Väänänen-Vainio-Mattila, K. The secret life of my dog: design and evaluation of paw tracker concept. In Proc. MobileHCI '11, ACM Press (2011), 231-240. [19] Sanders, C. R. The impact of guide dogs on the identity of people with visual impairments. Anthrozoös: A Multidisciplinary Journal of The Interactions of People and Animals, 13(3), (2000), 131-139.

[20] Seko, K. \& Fukuchi, K. A guidance technique for motion tracking with a handheld camera using auditory feedback. In Proc. UIST'12. ACM Press (2012), 95-96. [21] Väätäjä, H. \& Pesonen, E. Ethical issues and guidelines when conducting $\mathrm{HCI}$ studies with animals. In Proc. CHI EA '13. ACM Press (2013), 2159-2168. [22] Weilenmann, A., \& Juhlin, O. Understanding people and animals: the use of a positioning system in ordinary human-canine interaction. In Proc. CHI '11, ACM Press (2011), 2631-2640.

[23] Whitmarsh, L. The Benefits of Guide Dog Ownership Visual Impairment Research, 7(1), (2005), 27-42.

[24] Wiggett-Barnard, C., \& Steel, H. The experience of owning a guide dog. Disability \& Rehabilitation, 30(14), (2008), 1014-1026.

[25] Wingrave, C., Rose, J., Langston, T. \& LaViola, J. Early explorations of CAT: Canine Amusement\&Training. In Proc. CHI EA'10, ACM Press (2010), 2661-2669. [26] Yonezawa, K., Miyaki, T., \& Rekimoto, J. Cat@Log: sensing device attachable to pet cats for supporting human-pet interaction. In Proc. ACE '09, ACM Press (2009), 149-156. 